\title{
Non-escaping points of Zorich maps
}

\author{
Walter Bergweiler and Jie Ding
}

\begin{abstract}
We extend results about the dimension of the radial Julia set of certain exponential functions to quasiregular Zorich maps in higher dimensions. Our results improve on previous estimates of the dimension also in the special case of exponential functions.
\end{abstract}

\section{Introduction}

The Julia set $J(f)$ of an entire function $f$ is the set where the iterates $f^{n}$ of $f$ do not form a normal family and the escaping set $I(f)$ consists of all points which tend to infinity under iteration of $f$. These sets play a fundamental role in the iteration theory of entire functions. A result of Eremenko [6] says that $J(f)=\partial I(f)$. We refer to [2, 15] for an introduction to the iteration theory of entire functions.

We consider the exponential family consisting of the functions $E_{\lambda}(z):=$ $\lambda e^{z}$ with $\lambda \in \mathbb{C} \backslash\{0\}$. If $0<\lambda<1 / e$, then $E_{\lambda}$ has an attracting fixed point. Devaney and Krych [5] showed that then $J\left(E_{\lambda}\right)$ is equal to the complement of the attracting basin of this fixed point and $J\left(E_{\lambda}\right)$ consists of uncountably many pairwise disjoint curves (called hairs) which connect a finite point (called the endpoint of the hair) with $\infty$. Let $C_{\lambda}$ be the set of endpoints of the hairs that form $J\left(E_{\lambda}\right)$. The results of Devaney and Krych also yield that $J\left(E_{\lambda}\right) \backslash C_{\lambda} \subset I\left(E_{\lambda}\right)$.

McMullen [13] showed that $\operatorname{dim} J\left(E_{\lambda}\right)=2$. Here and in the following $\operatorname{dim} X$ denotes the Hausdorff dimension of a set $X$. In fact, McMullen showed that $\operatorname{dim} I\left(E_{\lambda}\right)=2$ and $I\left(E_{\lambda}\right) \subset J\left(E_{\lambda}\right)$. Karpińska [11] obtained the surprising result that $\operatorname{dim} J\left(E_{\lambda}\right) \backslash C_{\lambda}=1$.

A 3-dimensional analogue of the results of Devaney and Krych, McMullen and Karpińska was obtained in [3]. Here the exponential function was replaced by a quasiregular map $F: \mathbb{R}^{3} \rightarrow \mathbb{R}^{3}$ introduced by Zorich [19, p. 400]. 
As noted in [12, $\S 8.1]$, Zorich maps exist in $\mathbb{R}^{d}$ for all $d \geq 2$, and this can be used (see [3, Remark 9] and [4]) to obtain a $d$-dimensional analogue of the above results.

To define a Zorich map, following [9, §6.5.4], we fix $\rho>0$ and consider the cube

$$
Q:=\left\{x \in \mathbb{R}^{d-1}:\|x\|_{\infty} \leq \rho\right\}=[-\rho, \rho]^{d-1}
$$

and the upper hemisphere

$$
U:=\left\{x \in \mathbb{R}^{d}:\|x\|_{2}=1, x_{d} \geq 0\right\} .
$$

Let $h: Q \rightarrow U$ be a bi-Lipschitz map and define

$$
F: Q \times \mathbb{R} \rightarrow \mathbb{R}^{d}, F\left(x_{1}, \ldots, x_{d}\right)=e^{x_{d}} h\left(x_{1}, \ldots, x_{d-1}\right) .
$$

The map $F$ is then extended to a map $F: \mathbb{R}^{d} \rightarrow \mathbb{R}^{d}$ by repeated reflection at hyperplanes.

The main result of [3] says that if $a \in \mathbb{R}$ is sufficiently large, then the map

$$
f_{a}: \mathbb{R}^{d} \rightarrow \mathbb{R}^{d}, f_{a}(x)=F(x)-(0, \ldots, 0, a),
$$

has an attracting fixed point $\xi_{a}$ such that the complement of the attracting basin of $\xi_{a}$ consists of hairs, the set of endpoints of the hairs has dimension $d$, but the union of the hairs without the endpoints has dimension 1 .

The purpose of this paper is to extend some further results about the exponential family to the higher dimensional setting. Let $J_{\mathrm{bd}}\left(E_{\lambda}\right)$ be the set of all $z \in J\left(E_{\lambda}\right)$ for which the orbit $\left\{E_{\lambda}^{n}(z): n \in \mathbb{N}\right\}$ is bounded. Karpińska [10, Theorem 2] also showed that $\operatorname{dim} J_{\mathrm{bd}}\left(E_{\lambda}\right)>1$ for all $\lambda \in(0,1 / e)$ and

$$
1+\frac{1}{\log \log (1 / \lambda)}<\operatorname{dim} J_{\mathrm{bd}}\left(E_{\lambda}\right)<1+\frac{1}{\log \log \log (1 / \lambda)}
$$

if $\lambda$ is sufficiently small.

Urbański and Zdunik [16] considered the set $J_{\mathrm{r}}\left(E_{\lambda}\right):=J\left(E_{\lambda}\right) \backslash I\left(E_{\lambda}\right)$. We note that, in general, the notation $J_{\mathrm{r}}(f)$ is used for the radial Julia set of an entire function $f$, but for the functions $E_{\lambda}$ with $0<\lambda<1 / e$ this agrees with the above definition; see [14] for a discussion of radial Julia sets. Clearly $J_{\mathrm{r}}\left(E_{\lambda}\right) \supset J_{\mathrm{bd}}\left(E_{\lambda}\right)$. Urbański and Zdunik proved [16, Theorem 4.5] that $\operatorname{dim} J_{\mathrm{r}}\left(E_{\lambda}\right)=\operatorname{dim} J_{\mathrm{bd}}\left(E_{\lambda}\right)<2$ for $0<\lambda<1 / e$. They noted that (1.3) thus yields that

$$
\lim _{\lambda \rightarrow 0} \operatorname{dim} J_{\mathrm{r}}\left(E_{\lambda}\right)=1
$$


but they also gave a direct proof of this [16, Theorem 7.2].

Urbański and Zdunik also showed that the function $\lambda \mapsto \operatorname{dim} J_{\mathrm{r}}\left(E_{\lambda}\right)$ is continuous [16, Theorem 4.7] in the interval $(0,1 / e)$ and in fact realanalytic [17, Theorem 9.3]. The function $\lambda \mapsto \operatorname{dim} J_{\mathrm{r}}\left(E_{\lambda}\right)$, and in particular its behavior as $\lambda \rightarrow 1 / e$, was further studied in [8, 18].

We consider the corresponding sets for the Zorich maps. Denoting by $\xi_{a}$ the attracting fixed point of $f_{a}$ we thus put

$$
J_{\mathrm{bd}}\left(f_{a}\right):=\left\{x \in \mathbb{R}^{d}: f_{a}^{n}(x) \not \rightarrow \xi_{a} \text { and }\left(f_{a}^{n}(x)\right) \text { is bounded }\right\}
$$

and

$$
J_{\mathrm{r}}\left(f_{a}\right):=\left\{x \in \mathbb{R}^{d}: f_{a}^{n}(x) \not \rightarrow \xi_{a} \text { and }\left|f_{a}^{n}(x)\right| \not \rightarrow \infty\right\}
$$

Theorem 1.1. If a is sufficiently large, then

$d-1+\frac{1}{2} \frac{\log \log a}{\log a}-\frac{\log \log \log a}{\log a}<\operatorname{dim} J_{\mathrm{bd}}\left(f_{a}\right) \leq \operatorname{dim} J_{\mathrm{r}}\left(f_{a}\right) \leq d-1+\frac{\log \log a}{\log a}$.

It follows from Theorem 1.1 that if $0<\eta<\frac{1}{2}$ and $a$ is sufficiently large, then

$$
d-1+\eta \frac{\log \log a}{\log a}<\operatorname{dim} J_{\mathrm{bd}}\left(f_{a}\right),
$$

but this does not hold for $\eta=1$. It remains open for which $\eta \in\left[\frac{1}{2}, 1\right)$ the inequality (1.5) holds.

In order to compare Theorem 1.1 with (1.3) we note that for $d=2$, $\rho=\pi / 2$,

$$
h:\left[-\frac{\pi}{2}, \frac{\pi}{2}\right] \rightarrow \mathbb{R}^{2}=\mathbb{C}, h(x)=(\sin x, \cos x)=\sin x+i \cos x=i e^{-i x},
$$

and $z=(x, y)=x+i y$ the Zorich map $F$ takes the form

$$
F(z)=F(x, y)=e^{y} h(x)=i e^{y-i x}=i e^{-i z} .
$$

Hence for $a>0$ and $\lambda=e^{-a}$ we have

$$
f_{a}(z)=F(z)-i a=i\left(e^{-i z}-a\right)=\left(L \circ E_{\lambda} \circ L^{-1}\right)(z)
$$


with $L(z)=i(z-a)$. Thus $f_{a}$ is conjugate to $E_{\lambda}$. Since $a=\log (1 / \lambda)$ we see that Theorem 1.1 not only extends the results for the functions $E_{\lambda}$ to higher dimensions, but also improves (1.3) and (1.4) to

$$
\begin{gathered}
d-1+\frac{1}{2} \frac{\log \log \log (1 / \lambda)}{\log \log (1 / \lambda)}-\frac{\log \log \log \log (1 / \lambda)}{\log \log (1 / \lambda)} \\
<\operatorname{dim} J_{\mathrm{bd}}\left(E_{\lambda}\right) \leq \operatorname{dim} J_{\mathrm{r}}\left(E_{\lambda}\right) \leq 1+\frac{\log \log \log (1 / \lambda)}{\log \log (1 / \lambda)}
\end{gathered}
$$

Acknowledgment. This research was initiated while the second author was visiting the University of Kiel and it was completed while the first author was visiting the Shanghai Center of Mathematical Sciences. Both authors thank the respective institutions for the hospitality.

We also thank the referee for a large number of helpful remarks, in particular for suggesting an improvement of the lower bound in Theorem 1.1.

\section{Preliminaries}

We collect some results about Zorich maps which can be found in [3, 4]. As mentioned above, in [3] only the case $d=3$ is treated, but the changes to handle the general case are minor. We also note that in [3, 4] only the case $\rho=1$ is considered. However, it was noted already in [3, Remark 1] that one may replace the unit cube by a cube of other sidelength and in fact by a rectangular box. The reason that we do not restrict to the case $\rho=1$ is that this way the exponential map is (conjugate to) a special Zorich map, as described in the introduction.

We will use $|x|$ for the Euclidean norm of a point $x \in \mathbb{R}^{d}$; that is, we write $|x|=\|x\|_{2}$. For $c \in \mathbb{R}$ we define the half-space

$$
H_{>c}:=\left\{\left(x_{1}, \ldots, x_{d}\right) \in \mathbb{R}^{d}: x_{d}>c\right\} .
$$

The half-spaces $H_{<c}, H_{\geq c}$ and $H_{\leq c}$ and the hyperplane $H_{=c}$ are defined analogously.

First we note that the derivative $D F(x)$ exists almost everywhere and that if $D F\left(x_{1}, \ldots, x_{d-1}, 0\right)$ exists, then

$$
D F\left(x_{1}, \ldots, x_{d}\right)=e^{x_{d}} D F\left(x_{1}, \ldots, x_{d-1}, 0\right) .
$$


This implies that there exist $\alpha, m, M \in \mathbb{R}$ with $0<\alpha<1$ and $m<M$ such that

$$
|D F(x)|:=\sup _{|h|=1}|D F(x)(h)| \leq \alpha \quad \text { a.e. for } x \in H_{\leq m}
$$

while

$$
\ell(D F(x)):=\inf _{|h|=1}|D F(x)(h)| \geq \frac{1}{\alpha} \quad \text { a.e. for } x \in H_{\geq M} .
$$

We may and will assume that $M \geq 0$. It was shown in [3] that if

$$
a \geq e^{M}-m
$$

then $f_{a}$ has an attracting fixed point $\xi_{a} \in H_{\leq m}$ and the properties mentioned in the introduction hold; that is, the complement of the basin of attraction of $\xi_{a}$ consists of hairs, the set of endpoints of the hairs has dimension $d$, and the union of the hairs without endpoints has dimension 1.

The following result can be considered as an analogue of the result of Karpińska [10, Theorem 2] that $\operatorname{dim} J_{\mathrm{bd}}\left(E_{\lambda}\right)>1$ for $0<\lambda<1 / e$.

Theorem 2.1. If a satisfies (2.3), then $\operatorname{dim} J_{\mathrm{bd}}\left(f_{a}\right)>d-1$.

Theorem 2.1 will be proved in Section 4 together with the lower bound in Theorem 1.1 .

Remark 2.2. Urbański and Zdunik [16, Corollary 7.3] showed that (1.4) implies that $\operatorname{dim} J_{\mathrm{r}}\left(E_{\lambda}\right)<2$ whenever $0<\lambda<1 / e$ (and in fact whenever $E_{\lambda}$ has an attracting fixed point). The proof uses that if two functions in the exponential family both have an attracting fixed point, then they are quasiconformally conjugate. This argument is not available in the higherdimensional setting. We do not know whether $\operatorname{dim} J_{\mathrm{r}}\left(f_{a}\right)<d$ whenever $a$ satisfies (2.3).

For $r=\left(r_{1}, \ldots, r_{d-1}\right) \in \mathbb{Z}^{d-1}$ we put

$$
P(r):=\left\{\left(x_{1}, \ldots, x_{d-1}\right) \in \mathbb{R}^{d-1}:\left|x_{j}-2 \rho r_{j}\right|<\rho \text { for } 1 \leq j \leq d-1\right\}
$$

so that $P(0)$ is the interior of $Q$. Let

$$
S:=\left\{r \in \mathbb{Z}^{d-1}: \sum_{j=1}^{d-1} r_{j} \text { is even }\right\}
$$


Then $F$ maps $P(r) \times \mathbb{R}$ onto $H_{>0}$ if $r \in S$ and onto $H_{<0}$ if $r \in \mathbb{Z}^{d-1} \backslash S$. Thus $f_{a}$ maps $P(r) \times \mathbb{R}$ onto $H_{>-a}$ if $r \in S$ and onto $H_{<-a}$ if $r \in \mathbb{Z}^{d-1} \backslash S$. For $r \in S$ we put

$$
T(r):=P(r) \times(M, \infty)
$$

A short computation (see [3, (2.1)] or [4, (2.2)]) shows that $f_{a}(T(r)) \supset H_{\geq M}$. Thus there exists a branch $\Lambda^{r}: H_{\geq M} \rightarrow T(r)$ of the inverse function of $f_{a}$. With $\Lambda:=\Lambda^{(0, \ldots, 0)}$ we have

$$
\Lambda^{\left(r_{1}, \ldots, r_{d-1}\right)}(x)=\Lambda(x)+\left(2 \rho r_{1}, \ldots, 2 \rho r_{d-1}, 0\right)
$$

for all $x \in H_{\geq M}$ and all $r \in S$. We have

$$
D \Lambda(x)=D f_{a}(\Lambda(x))^{-1}=D F(\Lambda(x))^{-1} \quad \text { a.e. for } x \in H_{\geq M} .
$$

It thus follows from (2.2) that

$$
|D \Lambda(x)| \leq \alpha \quad \text { a.e. for } x \in H_{\geq M}
$$

This implies that

$$
|\Lambda(x)-\Lambda(y)| \leq \alpha|x-y| \text { for } x, y \in H_{\geq M}
$$

Noting that $D f_{a}(x)=D F(x)$ we deduce from (2.1) that there exist positive constants $c_{1}$ and $c_{2}$ such that

$$
c_{1} e^{x_{d}} \leq \ell\left(D f_{a}(x)\right) \leq\left|D f_{a}(x)\right| \leq c_{2} e^{x_{d}} \quad \text { a.e. }
$$

It was shown in [3, 4] that there exists positive constants $c_{3}$ and $c_{4}$ such that

$$
\frac{c_{3}}{|x|} \leq \ell(D \Lambda(x)) \leq|D \Lambda(x)| \leq \frac{c_{4}}{|x|} \quad \text { a.e. for } x \in H_{\geq M}
$$

and this was used to prove that

$$
|\Lambda(x)-\Lambda(y)| \leq c_{4} \pi \frac{|x-y|}{\min \{|x|,|y|\}} .
$$

We have to consider how the bounds for $\ell(D \Lambda(x))$ and $|D \Lambda(x)|$ in (2.7) depend on $a$. We will write $\bar{a}=(0, \ldots, 0, a)$ so that $f_{a}(x)=F(x)-\bar{a}$. 
Lemma 2.3. There exist constants $c_{3}$ and $c_{4}$ depending only on $F$ such that if a satisfies (2.3), then

$$
\frac{c_{3}}{|x+\bar{a}|} \leq \ell(D \Lambda(x)) \leq|D \Lambda(x)| \leq \frac{c_{4}}{|x+\bar{a}|} \quad \text { a.e. for } x \in H_{\geq M} \text {. }
$$

Proof. By (2.4), (2.6), (1.1) and (1.2) we have

$$
\begin{aligned}
|D \Lambda(x)| & \leq \frac{1}{\ell(D F(\Lambda(x)))} \leq \frac{1}{c_{1} \exp \left(\Lambda_{d}(x)\right)} \\
& =\frac{1}{c_{1}|F(\Lambda(x))|}=\frac{1}{c_{1}\left|f_{a}(\Lambda(x))+\bar{a}\right|}=\frac{1}{c_{1}|x+\bar{a}|} .
\end{aligned}
$$

The proof of the lower bound for $\ell(D \Lambda(x))$ is similar.

Lemma 2.3 implies that (2.8) can be improved to

$$
|\Lambda(x)-\Lambda(y)| \leq c_{4} \pi \frac{|x-y|}{\min \{|x+\bar{a}|,|y+\bar{a}|\}}
$$

for $x, y \in H_{\geq M}$.

\section{Proof of the upper bound in Theorem 1.1}

For $r \in S$ and $A \subset \mathbb{R}^{d}$, we will use the notation

$$
\begin{aligned}
A^{r} & :=\left(2 \rho r_{1}, \ldots, 2 \rho r_{d-1}, 0\right)+A \\
& =\left\{\left(2 \rho r_{1}+x_{1}, \ldots, 2 \rho r_{d-1}+x_{d-1}, x_{d}\right): x \in A\right\} .
\end{aligned}
$$

We also write $B(x, R)$ for the closed ball of radius $R$ around a point $x \in \mathbb{R}^{d}$. We note that since $M>m$ it follows from (2.3) that $a>1$.

Lemma 3.1. Let $r, s \in S$ and let $A \subset T(s)$ be bounded. If a satisfies (2.3), then

$$
\operatorname{diam} \Lambda\left(A^{r}\right) \leq c_{4} \pi \frac{\operatorname{diam} A}{\sqrt{\rho^{2}|r+s|^{2}+a^{2}}} .
$$

Proof. Since $A^{r} \subset T(r+s)$ we find that if $x=\left(x_{1}, \ldots, x_{d}\right) \in A^{r}$, then $\left|x_{j}\right| \geq \max \left\{2 \rho\left|r_{j}+s_{j}\right|-\rho, 0\right\} \geq \rho\left|r_{j}+s_{j}\right|$ for $1 \leq j \leq d-1$ while $x_{d} \geq M$. 
Thus, recalling that $M \geq 0$, we find that

$$
\begin{aligned}
|x+\bar{a}| & =\sqrt{\sum_{j=1}^{d-1} x_{j}^{2}+\left(x_{d}+a\right)^{2}} \\
& \geq \sqrt{\sum_{j=1}^{d-1} \rho^{2}\left(r_{j}+s_{j}\right)^{2}+a^{2}}=\sqrt{\rho^{2}|r+s|^{2}+a^{2}} .
\end{aligned}
$$

The conclusion now follows from (2.9).

Lemma 3.2. There exist positive constants $c_{5}$ and $c_{6}$ depending only on $d$ such that if $d-1<t \leq d$ and $N \geq b \geq 3 \sqrt{d-1}$, then

$$
c_{5} \frac{b^{d-1-t}}{t-d+1}\left(1-\left(\frac{N}{b}\right)^{d-1-t}\right) \leq \sum_{\substack{r \in S \\|r| \leq N}} \frac{1}{\left(|r|^{2}+b^{2}\right)^{t / 2}} \leq c_{6} \frac{b^{d-1-t}}{t-d+1} .
$$

Moreover,

$$
\sum_{\substack{r \in S \\|r| \leq N}} \frac{1}{\left(|r|^{2}+b^{2}\right)^{(d-1) / 2}} \geq c_{5} \log \frac{N}{b}
$$

Proof. For $r=\left(r_{1}, \ldots, r_{d-1}\right) \in \mathbb{Z}^{d-1}$ let $Q_{r}$ be the cube with vertices at the points $\left(2 r_{1}+e_{1}, \ldots, 2 r_{d-1}+e_{d-1}\right)$, where $e_{j} \in\{-1,1\}$ for all $j$. For $x \in Q_{r}$ and $b \geq \sqrt{d-1} / 7$ we then have

$$
|x|^{2} \leq \sum_{j=1}^{d-1}\left(2\left|r_{j}\right|+1\right)^{2} \leq \sum_{j=1}^{d-1}\left(8 r_{j}^{2}+1\right)=8|r|^{2}+d-1 \leq 8|r|^{2}+7 b^{2}
$$

and thus $|x|^{2}+b^{2} \leq 8|r|^{2}+8 b^{2}$. Hence

$$
\int_{Q_{r}} \frac{d x_{1} \ldots d x_{d-1}}{\left(|x|^{2}+b^{2}\right)^{t / 2}} \geq \int_{Q_{r}} \frac{d x_{1} \ldots d x_{d-1}}{\left(8|r|^{2}+8 b^{2}\right)^{t / 2}}=\frac{2^{d-1} 8^{-t / 2}}{\left(|r|^{2}+b^{2}\right)^{t / 2}}=\frac{2^{d-1-3 t / 2}}{\left(|r|^{2}+b^{2}\right)^{t / 2}} .
$$


For $|r| \leq N$ we have $Q_{r} \subset B(0,2 N+\sqrt{d-1}) \subset B(0,2 N+d)$ and thus

$$
\begin{aligned}
\sum_{\substack{r \in S \\
|r| \leq N}} \frac{1}{\left(|r|^{2}+b^{2}\right)^{t / 2}} & \leq 2^{3 t / 2-d+1} \sum_{\substack{r \in S \\
|r| \leq N}} \int_{Q_{r}} \frac{d x_{1} \ldots d x_{d-1}}{\left(|x|^{2}+b^{2}\right)^{t / 2}} \\
& \leq 2^{3 t / 2-d+1} \int_{B(0,2 N+d)} \frac{d x_{1} \ldots d x_{d-1}}{\left(|x|^{2}+b^{2}\right)^{t / 2}} \\
& =2^{3 t / 2-d+1} \frac{2 \pi^{(d-1) / 2}}{\Gamma\left(\frac{d-1}{2}\right)} \int_{0}^{2 N+d} \frac{u^{d-2}}{\left(u^{2}+b^{2}\right)^{t / 2}} d u .
\end{aligned}
$$

Furthermore,

$$
\begin{aligned}
\int_{0}^{2 N+d} \frac{u^{d-2}}{\left(u^{2}+b^{2}\right)^{t / 2}} d u & =b^{d-1-t} \int_{0}^{(2 N+d) / b} \frac{v^{d-2}}{\left(v^{2}+1\right)^{t / 2}} d v \\
& \leq b^{d-1-t}\left(1+\int_{1}^{\infty} v^{d-2-t} d v\right) \\
& =b^{d-1-t}\left(1+\frac{1}{t-d+1}\right) \leq 2 \frac{b^{d-1-t}}{t-d+1}
\end{aligned}
$$

The right inequality in (3.1) now follows from (3.3) and (3.4).

To prove the left inequality, we proceed similarly. For $r \in S$ let $P_{r}$ be the cube with vertices at the points $\left(2 r_{1}+3 e_{1}, \ldots, 2 r_{d-1}+3 e_{d-1}\right)$, where $e_{j} \in\{-1,1\}$ for all $j$. Noting that $(2 y-3)^{2} \geq y^{2}-3$ for $y \in \mathbb{R}$ we find that if $x \in P_{r}$ and $b \geq 3 \sqrt{d-1}$, then

$$
|x|^{2} \geq \sum_{j=1}^{d-1}\left(2\left|r_{j}\right|-3\right)^{2} \geq \sum_{j=1}^{d-1}\left(\left|r_{j}\right|^{2}-3\right)=|r|^{2}-3(d-1) \geq|r|^{2}-\frac{1}{2} b^{2}
$$

and thus $|x|^{2}+b^{2} \geq \frac{1}{2}|r|^{2}+\frac{1}{2} b^{2}$. Hence

$$
\int_{P_{r}} \frac{d x_{1} \ldots d x_{d-1}}{\left(|x|^{2}+b^{2}\right)^{t / 2}} \leq \int_{P_{r}} \frac{d x_{1} \ldots d x_{d-1}}{\left(\frac{1}{2}|r|^{2}+\frac{1}{2} b^{2}\right)^{t / 2}}=\frac{6^{d-1} 2^{t / 2}}{\left(|r|^{2}+b^{2}\right)^{t / 2}}
$$

For $x \in \mathbb{R}^{d-1}$ we can choose $r \in S$ such that $x \in P_{r}$. In fact, we first choose $r \in \mathbb{Z}^{d-1}$ such that $\left|x_{j}-2 r_{j}\right| \leq 1$ for all $j$, and in order to achieve that $\sum_{j=1}^{d-1} r_{j}$ is even we replace $r_{1}$ by $r_{1}+1$ if necessary. For $x \in P_{r}$ we have 
$\left|r_{j}\right| \leq\left(\left|x_{j}\right|+3\right) / 2$ and since $N \geq 3 \sqrt{d-1}$ this implies that

$$
\begin{aligned}
|r|^{2} & =\sum_{j=1}^{d-1} r_{j}^{2} \leq \frac{1}{4} \sum_{j=1}^{d-1}\left(\left|x_{j}\right|+3\right)^{2} \leq \frac{1}{4} \sum_{j=1}^{d-1} 2\left(x_{j}^{2}+9\right) \\
& =\frac{1}{2}|x|^{2}+\frac{9}{2}(d-1) \leq \frac{1}{2}|x|^{2}+\frac{1}{2} N^{2} .
\end{aligned}
$$

For $|x| \leq N$ we thus have $|r| \leq N$ so that

$$
\bigcup_{\substack{r \in S \\|r| \leq N}} P_{r} \supset B(0, N) .
$$

Instead of (3.3) we now obtain

$$
\begin{aligned}
\sum_{\substack{r \in S \\
|r| \leq N}} \frac{1}{\left(|r|^{2}+b^{2}\right)^{t / 2}} & \geq 6^{1-d} 2^{-t / 2} \sum_{\substack{r \in S \\
|r| \leq N}} \int_{P_{r}} \frac{d x_{1} \ldots d x_{d-1}}{\left(|x|^{2}+b^{2}\right)^{t / 2}} \\
& \geq 6^{1-d} 2^{-t / 2} \int_{B(0, N)} \frac{d x_{1} \ldots d x_{d-1}}{\left(|x|^{2}+b^{2}\right)^{t / 2}} \\
& =6^{1-d} 2^{-t / 2} \frac{2 \pi^{(d-1) / 2}}{\Gamma\left(\frac{d-1}{2}\right)} \int_{0}^{N} \frac{u^{d-2}}{\left(u^{2}+b^{2}\right)^{t / 2}} d u
\end{aligned}
$$

and instead of (3.4) we have

$$
\begin{aligned}
\int_{0}^{N} \frac{u^{d-2}}{\left(u^{2}+b^{2}\right)^{t / 2}} d u & =b^{d-1-t} \int_{0}^{N / b} \frac{v^{d-2}}{\left(v^{2}+1\right)^{t / 2}} d v \\
& \geq b^{d-1-t} 2^{-t / 2} \int_{1}^{N / b} v^{d-2-t} d v \\
& =\frac{b^{d-1-t}}{t-d+1} 2^{-t / 2}\left(1-\left(\frac{N}{b}\right)^{d-1-t}\right) .
\end{aligned}
$$

The left inequality in (3.1) now follows from (3.5) and (3.6).

Finally, to prove $(\underline{3.2})$ we only have to note that for $t=d-1$ we obtain

$$
\begin{aligned}
\int_{0}^{N} \frac{u^{d-2}}{\left(u^{2}+b^{2}\right)^{(d-1) / 2}} d u & =\int_{0}^{N / b} \frac{v^{d-2}}{\left(v^{2}+1\right)^{(d-1) / 2}} d v \\
& \geq 2^{(1-d) / 2} \int_{1}^{N / b} \frac{d v}{v}=2^{(1-d) / 2} \log \frac{N}{b}
\end{aligned}
$$

instead of (3.6). 
Lemma 3.3. There exists a constant $c_{7}$, depending only on $F$, such that if a satisfies (2.3) $, d-1<t \leq d, s \in S$ and $A \subset T(s)$ is bounded, then

$$
\sum_{r \in S}\left(\operatorname{diam} \Lambda\left(A^{r}\right)\right)^{t} \leq c_{7} \frac{a^{d-1-t}}{t-d+1}(\operatorname{diam} A)^{t} .
$$

Proof. Without loss of generality we may assume that $s=0$. Applying Lemma 3.1 we obtain

$$
\begin{aligned}
\sum_{r \in S}\left(\operatorname{diam} \Lambda\left(A^{r}\right)\right)^{t} & \leq \sum_{r \in S} \frac{\left(c_{4} \pi \operatorname{diam} A\right)^{t}}{\left(\rho^{2}|r|^{2}+a^{2}\right)^{t / 2}} \\
& \leq\left(\frac{c_{4} \pi \operatorname{diam} A}{\rho}\right)^{t} \sum_{r \in S} \frac{1}{\left(|r|^{2}+(a / \rho)^{2}\right)^{t / 2}}
\end{aligned}
$$

We note that the upper bound in (3.1) does not depend on $N$. Thus we may take the limit as $N \rightarrow \infty$ there, which together with (3.7) yields that

$$
\begin{aligned}
\sum_{r \in S}\left(\operatorname{diam} \Lambda\left(A^{r}\right)\right)^{t} & \leq\left(\frac{c_{4} \pi}{\rho}\right)^{t} \frac{c_{6}}{t-d+1}\left(\frac{a}{\rho}\right)^{d-1-t}(\operatorname{diam} A)^{t} \\
& =\frac{c_{6}\left(c_{4} \pi\right)^{t}}{\rho^{d-1}} \frac{a^{d-1-t}}{t-d+1}(\operatorname{diam} A)^{t} .
\end{aligned}
$$

The conclusion follows.

Proof of the upper bound in Theorem 1.1. Let $J\left(f_{a}\right):=\left\{x: f_{a}^{n}(x) \not \rightarrow \xi_{a}\right\}$ be the complement of the attracting basin of $\xi_{a}$. For $R>M$ we consider the set

$$
K(R):=\left\{x \in J\left(f_{a}\right) \cap B(0, R): \liminf _{k \rightarrow \infty}\left|f_{a}^{k}(x)\right|<R\right\} .
$$

Let $c_{7}$ be the constant from Lemma 3.3. We show that if $a$ is sufficiently large and $d-1<t \leq d$ such that $\tau:=c_{7} a^{d-1-t} /(t-d+1)<1$, then $\operatorname{dim} K(R) \leq t$. This implies that $\operatorname{dim} J_{\mathrm{r}}\left(f_{a}\right) \leq t$, since $J_{\mathrm{r}}\left(f_{a}\right)=\bigcup_{n \in \mathbb{N}} K\left(R_{n}\right)$ for any sequence $\left(R_{n}\right)$ which tends to $\infty$. The conclusion follows from this, since for $t=d-1+\log \log a / \log a$ we have $a^{d-1-t} /(t-d+1)=1 / \log \log a$.

For $s \in S, A \subset T(s)$ and $n \in \mathbb{N}$, let $X_{n}(A)$ denote the set of all components of $f^{-n}(A)$ which are contained in $T(0)$. If $U \in X_{n}(A)$, then $f(U)$ has the form $f(U)=V^{r}$ for some $V \in X_{n-1}(A)$ and some $r \in S$. Equivalently, 
$U=\Lambda\left(V^{r}\right)$. In turn, if $V \in X_{n-1}(A)$ and $r \in S$, then $\Lambda\left(V^{r}\right) \in X_{n}(A)$. Together with Lemma 3.3 we thus find that

$$
\sum_{U \in X_{n}(A)}(\operatorname{diam} U)^{t}=\sum_{V \in X_{n-1}(A)} \sum_{r \in S}\left(\operatorname{diam} \Lambda\left(V^{r}\right)\right)^{t} \leq \tau \sum_{V \in X_{n-1}(A)}(\operatorname{diam} V)^{t} .
$$

Induction yields that

$$
\begin{aligned}
\sum_{U \in X_{n}(A)}(\operatorname{diam} U)^{t} & \leq \tau^{n-1} \sum_{V \in X_{1}(A)}(\operatorname{diam} V)^{t} \\
& =\tau^{n-1}(\operatorname{diam} \Lambda(A))^{t} \leq \tau^{n-1}(\operatorname{diam} A)^{t} .
\end{aligned}
$$

We will apply this for $A^{s}:=H_{\leq R} \cap T(s)$. There exists $N \in \mathbb{N}$ such that

$$
J\left(f_{a}\right) \cap B(0, R) \subset \bigcup_{\substack{s \in S \\|s| \leq N}} A^{s}
$$

Next we put

$$
Y_{n}:=\bigcup_{\substack{s \in S \\|s| \leq N}} \bigcup_{m \geq n} X_{m}\left(A^{s}\right) \text { and } Z_{n}:=\left\{U^{s}: s \in S,|s| \leq N, U \in Y_{n}\right\} .
$$

Then $Y_{n}$ contains all points $x \in T(0)$ for which there exists $m \geq n$ and $s \in S$ with $|s| \leq N$ such that $f_{a}^{m}(x) \in A^{s}$. Hence $Z_{n}$ contains all $x \in \bigcup_{s \in S,|s| \leq N} T(s)$ for which there exists $m \geq n$ such that $f_{a}^{m}(x) \in \bigcup_{s \in S,|s| \leq N} A^{s}$. In particular, $Z_{n}$ contains all $x \in J\left(f_{a}\right) \cap B(0, R)$ for which there exists $m \geq n$ such that $\left|f_{a}^{m}(x)\right| \leq R$. Thus $K(R) \subset Z_{n}$ for all $n \in \mathbb{N}$.

Let $L$ be the cardinality of $\{s \in S:|s| \leq N\}$. Then

$$
\begin{aligned}
\sum_{U \in Z_{n}}(\operatorname{diam} U)^{t} & =L \sum_{U \in Y_{n}}(\operatorname{diam} U)^{t}=L \sum_{\substack{s \in S \\
|s| \leq N}} \sum_{m \geq n} \sum_{U \in X_{m}\left(A^{s}\right)}(\operatorname{diam} U)^{t} \\
& \leq L \sum_{\substack{s \in S \\
|s| \leq N}} \sum_{m \geq n} \tau^{m-1}\left(\operatorname{diam} A^{s}\right)^{t}=L^{2} \sum_{m \geq n} \tau^{m-1}\left(\operatorname{diam} A^{0}\right)^{t} \\
& =L^{2}\left(\operatorname{diam} A^{0}\right)^{t} \frac{\tau^{n-1}}{1-\tau}
\end{aligned}
$$

by (3.8). Since the right hand side tends to 0 as $n \rightarrow \infty$, we deduce that $\operatorname{dim} K(R) \leq t$. 


\section{Proof of the lower bounds}

The proof is based on the theory of iterated functions systems. A similar method was used in [1] to estimate the dimensions of Julia sets from below. In particular, we will use the following result [7, Proposition 9.7].

Lemma 4.1. Let $S_{1}, \ldots, S_{m}$ be contractions on a closed subset $K$ of $\mathbb{R}^{d}$ such that there exists $b_{1}, \ldots, b_{m} \in(0,1)$ with

$$
b_{j}|x-y| \leq\left|S_{j}(x)-S_{j}(y)\right| \quad \text { for } x, y \in K \text { and } 1 \leq j \leq m .
$$

Suppose that $K_{0}$ is a non-empty compact subset of $K$ with

$$
K_{0}=\bigcup_{j=1}^{m} S_{j}\left(K_{0}\right)
$$

and $S_{j}\left(K_{0}\right) \cap S_{k}\left(K_{0}\right)=\emptyset$ for $j \neq k$. Let $t>0$ with

$$
\sum_{j=1}^{m} b_{j}^{t}=1 .
$$

Then $\operatorname{dim} K_{0} \geq t$.

Since the left hand side of (4.1) is a decreasing function of $t$, it follows that if

$$
\sum_{j=1}^{m} b_{j}^{t}>1,
$$

then $\operatorname{dim} K_{0}>t$.

Proof of Theorem 2.1] and the lower bound in Theorem 1.1. Let $N \in \mathbb{N}$ with $N \geq a / \rho$ and put $R:=8 \rho N$ and $K:=B(-\bar{a}, R) \cap H_{\geq M}$. Note that if $N$ is sufficiently large, then $R>M-a$ so that $K$ is non-empty.

Next we note that if $x=\left(x_{1}, \ldots, x_{d}\right) \in \mathbb{R}^{d}$ with $x_{d}>\log R$, then

$$
|f(x)+a|=|f(x)|=e^{x_{d}}>R
$$

and thus $f(x) \notin B(-\bar{a}, R)$. It follows that

$$
\Lambda^{r}(K) \subset A^{r}:=H_{\leq \log R} \cap T(r)
$$


for $r \in S$.

Put $L:=a+\log R=a+\log (8 \rho N)$. For $y \in A^{r}$ we have

$$
|y+\bar{a}|^{2} \leq \sum_{j=1}^{d-1}\left(2\left|r_{j}\right|+1\right)^{2} \rho^{2}+L^{2} \leq 8 \rho^{2}|r|^{2}+(d-1) \rho^{2}+L^{2} \leq 8\left(\rho^{2}|r|^{2}+L^{2}\right)
$$

if $N$ and hence $L$ are large. Since $a \leq \rho N$ we have $L \leq \rho N+\log (8 \rho N) \leq 2 \rho N$ if $N$ and $L$ are large. Thus we see that if $|r| \leq N$ and $y \in A_{r}$, then

$$
|y+\bar{a}|^{2} \leq 8\left(\rho^{2} N^{2}+4 \rho^{2} N^{2}\right)=40 \rho^{2} N^{2} \leq R^{2} .
$$

Thus $A^{r} \subset K$ if $|r| \leq N$, provided $N$ is sufficiently large. Hence $\Lambda^{r}(K) \subset K$ if $r \in S$ and $|r| \leq N$. Together with (2.5) this implies that the $\Lambda^{r}$ are contractions on $K$.

It follows that the functions $S_{j}$ of the form $S_{j}=\Lambda^{r} \circ \Lambda^{s}$, where $r, s \in S$ and $|r|,|s| \leq N$, are also contractions and hence form an iterated function system on $K$. We will apply Lemma 4.1 to this iterated function system.

It follows from Lemma 2.3 that $\ell\left(D \Lambda^{r}(x)\right)=\ell(D \Lambda(x)) \geq c_{3} / R$ for $x \in K$ and $r \in S$. By (4.2) we also have

$$
\ell\left(D \Lambda^{s}(y)\right)=\ell(D \Lambda(y)) \geq \frac{c_{3}}{|y+\bar{a}|} \geq \frac{c_{3}}{2 \sqrt{2} \sqrt{\rho^{2}|r|^{2}+L^{2}}} \quad \text { for } y \in A^{r} .
$$

Hence

$$
\ell\left(D\left(\Lambda^{s} \circ \Lambda^{r}\right)(y)\right) \geq \ell\left(D \Lambda^{s}\left(\Lambda^{r}(x)\right)\right) \cdot \ell\left(D \Lambda^{r}(x)\right) \geq \frac{c_{3}^{2}}{2 \sqrt{2} R \sqrt{\rho^{2}|r|^{2}+L^{2}}}
$$

for $x \in K$. It follows that

$$
\left|\left(\Lambda^{s} \circ \Lambda^{r}\right)(x)-\left(\Lambda^{s} \circ \Lambda^{r}\right)(y)\right| \geq b_{r, s}|x-y|
$$

with

$$
b_{r, s}=\frac{c_{3}^{2}}{2 \sqrt{2} R \sqrt{\rho^{2}|r|^{2}+L^{2}}},
$$

for $|r|,|s| \leq N$ and $x, y \in K$. The limit set $K_{0}$ of the iterated function system generated by the functions $\Lambda^{s} \circ \Lambda^{r}$ is contained in $J_{\mathrm{bd}}\left(f_{a}\right)$. It thus follows from Lemma 4.1 and the remark following it that $\operatorname{dim} J_{\mathrm{bd}}\left(f_{a}\right)>t$ if

$$
\sum_{\substack{r \in S \\|r| \leq N}} \sum_{\substack{s \in S \\|s| \leq N}} b_{r, s}^{t}>1
$$


Let the cube $P_{r}$ be defined as in the proof of Lemma 3.2. Then each $P_{r}$ has volume $6^{d-1}$ and the union of all $P_{r}$ for which $|r| \leq N$ covers $B(0, N)$ and thus has volume at least $\pi^{(d-1) / 2} N^{d-1} / \Gamma((d+1) / 2)$. Thus the set of all $s \in S$ for which $|s| \leq N$ has at least $\left\lfloor 6^{1-d} \pi^{(d-1) / 2} N^{d-1} / \Gamma((d+1) / 2)\right\rfloor$ elements. Recalling that $R=8 \rho N$ we deduce that there exist a constant $c_{8}$ such that with $b:=L / \rho=(a+\log (8 \rho N)) / \rho$ we have

$$
\begin{aligned}
\sum_{\substack{r \in S \\
|r| \leq N}} \sum_{\substack{s \in S \\
|s| \leq N}} b_{r, s}^{t} & \geq\left\lfloor\frac{6^{1-d} \pi^{(d-1) / 2} N^{d-1}}{\Gamma\left(\frac{d+1}{2}\right)}\right\rfloor \frac{c_{3}^{2 t}}{(2 \sqrt{2} R)^{t}} \sum_{\substack{r \in S \\
|r| \leq N}} \frac{1}{\left(\rho^{2}|r|^{2}+L^{2}\right)^{t / 2}} \\
& \geq c_{8} N^{d-1-t} \sum_{\substack{r \in S \\
|r| \leq N}} \frac{1}{\left(|r|^{2}+b^{2}\right)^{t / 2}},
\end{aligned}
$$

for $d-1 \leq t \leq d$.

For large $N$ we have $N \geq b=(a+\log (8 \rho N)) / \rho \geq 3 \sqrt{d-1}$. Thus Lemma 3.2 is applicable.

Suppose first that $t=d-1$. Using (3.2) we find with $c_{9}:=c_{8} c_{5}$ that

$$
\sum_{\substack{r \in S \\|r| \leq N}} \sum_{\substack{s \in S \\|s| \leq N}} b_{r, s}^{d-1} \geq c_{9} \log \frac{N}{b}=c_{9} \log \frac{N \rho}{a+\log (8 \rho N)}
$$

The right hand side of (4.5) tends to $\infty$ as $N \rightarrow \infty$. In particular, it is greater than 1 for large $N$ so that (4.3) holds for $t=d-1$. Thus $\operatorname{dim} J_{\mathrm{bd}}\left(f_{a}\right)>d-1$. This proves Theorem 2.1.

Now we consider the behavior as $a \rightarrow \infty$. Let $t=d-1+\gamma(a)$ where

$$
\gamma(a):=\frac{1}{2} \frac{\log \log a}{\log a}-\frac{\log \log \log a}{\log a}
$$

Then $d-1<t \leq d$ for large $a$. We also put $\beta(a):=e^{1 / \gamma(a)}$ and note that $\beta(a) \rightarrow \infty$ as $a \rightarrow \infty$. We may choose $N$ such that $N \sim a \beta(a)$ as $a \rightarrow \infty$. Since $\log \beta(a)=1 / \gamma(a)$ we find that $L=a+\log (8 \rho N) \sim a$ and hence $b=L / \rho \sim a / \rho$.

With $C:=2 / \rho$ we thus have $N b \leq C a^{2} \beta(a)$ and $N / b \geq \beta(a) / C$ for large $a$. By the definition of $\beta(a)$ we have $\beta(a)^{\gamma(a)}=e$. For large $a$ we also 
have $\frac{1}{2} \leq C^{\gamma(a)} \leq 2$. It thus follows from (4.4) and Lemma 3.2 that

$$
\begin{aligned}
\sum_{\substack{r \in S \\
|r| \leq N}} \sum_{\substack{s \in S \\
|s| \leq N}} b_{r, s}^{t} & \geq c_{9} \frac{(N b)^{d-1-t}}{t-d+1}\left(1-\left(\frac{N}{b}\right)^{d-1-t}\right) \\
& \geq c_{9} \frac{\left(C a^{2} \beta(a)\right)^{-\gamma(a)}}{\gamma(a)}\left(1-\left(\frac{\beta(a)}{C}\right)^{-\gamma(a)}\right) \\
& \geq \frac{c_{9}}{2 e} \frac{a^{-2 \gamma(a)}}{\gamma(a)}\left(1-\frac{2}{e}\right)
\end{aligned}
$$

for large $a$. It is easy to see that $\log \gamma(a)=\log \log \log a-\log \log a+\mathcal{O}(1)$ as $a \rightarrow \infty$. It thus follows that

$$
\log \left(\frac{a^{-2 \gamma(a)}}{\gamma(a)}\right)=-2 \gamma(a) \log a-\log \gamma(a)=\log \log \log a+\mathcal{O}(1)
$$

as $a \rightarrow \infty$. Thus the right hand side of (4.6) tends to $\infty$ as $a \rightarrow \infty$. Hence (4.3) holds for $t=d-1+\gamma(a)$ and large $a$. This proves the lower bound in Theorem 1.1.

\section{References}

[1] Krzysztof Barański, Bogusława Karpińska and Anna Zdunik, Hyperbolic dimension of Julia sets of meromorphic maps with logarithmic tracts. Int. Math. Res. Not. IMRN 2009, no. 4, 615-624.

[2] Walter Bergweiler, Iteration of meromorphic functions. Bull. Amer. Math. Soc. (N.S.) 29 (1993), no. 2, 151-188.

[3] Walter Bergweiler, Karpińska's paradox in dimension 3. Duke Math. J. 154 (2010), no. 3, 599-630.

[4] Patrick Comdühr, On the differentiability of hairs for Zorich maps. Ergodic Theory Dynam. Systems 39 (2019), no. 7, 1824-1842.

[5] Robert L. Devaney and Michał Krych, Dynamics of $\exp (z)$. Ergodic Theory Dynam. Systems 4 (1984), no. 1, 35-52. 
[6] A. È. Erëmenko, On the iteration of entire functions. In "Dynamical systems and ergodic theory (Warsaw, 1986)", Banach Center Publications 23. Polish Scientific Publishers, Warsaw 1989, pp. 339-345.

[7] Kenneth Falconer, Fractal geometry. Mathematical foundations and applications. John Wiley \& Sons, Chichester, 1990.

[8] Guillaume Havard, Mariusz Urbański and Michel Zinsmeister, Variations of Hausdorff dimension in the exponential family. Ann. Acad. Sci. Fenn. Math. 35 (2010), no. 2, 351-378.

[9] Tadeusz Iwaniec and Gaven Martin, Geometric function theory and nonlinear analysis. Oxford Mathematical Monographs. Oxford University Press, New York, 2001.

[10] Bogusława Karpińska, Area and Hausdorff dimension of the set of accessible points of the Julia sets of $\lambda e^{z}$ and $\lambda \sin z$. Fund. Math. 159 (1999), no. 3, 269-287.

[11] Bogusława Karpińska, Hausdorff dimension of the hairs without endpoints for $\lambda \exp z$. C. R. Acad. Sci. Paris Sér. I Math. 328 (1999), no. 11, 1039-1044.

[12] Olli Martio and Uri Srebro, Periodic quasimeromorphic mappings in $R^{n}$. J. Anal. Math. 28 (1975), no. 1, 20-40.

[13] Curt McMullen, Area and Hausdorff dimension of Julia sets of entire functions. Trans. Amer. Math. Soc. 300 (1987), no. 1, 329-342.

[14] Lasse Rempe, Hyperbolic dimension and radial Julia sets of transcendental functions. Proc. Amer. Math. Soc. 137 (2009), no. 4, 1411-1420.

[15] Dierk Schleicher, Dynamics of entire functions. In "Holomorphic dynamical systems", Lecture Notes Math. 1998, Springer, Berlin, 2010, pp. 295-339.

[16] Mariusz Urbański and Anna Zdunik, The finer geometry and dynamics of the hyperbolic exponential family. Michigan Math. J. 51 (2003), no. 2, 227-250. 
[17] Mariusz Urbański and Anna Zdunik, Real analyticity of Hausdorff dimension of finer Julia sets of exponential family. Ergodic Theory Dynam. Systems 24 (2004), no. 1, 279-315.

[18] Mariusz Urbański and Anna Zdunik, The parabolic map $f_{1 / e}(z)=\frac{1}{e} e^{z}$. Indag. Math. (N.S.) 15 (2004), no. 3, 419-433.

[19] V. A. Zorich, A theorem of M. A. Lavrent'ev on quasiconformal space maps. Math. USSR, Sb. 3 (1967), no. 3, 389-403; translation from Mat. Sb. (N.S.) 74 (116), (1967), 417-433.

Mathematisches Seminar

Christian-Albrechts-Universität zu Kiel

Ludewig-Meyn-Str. 4

24098 Kiel, Germany

E-mail: bergweiler@math.uni-kiel.de

School of Mathematics

Taiyuan University of Technology

Taiyuan 030024

China

E-mail: dingjie@tyut.edu.cn 